\title{
Les rapports préoccupants des futurs enseignants avec les milieux physiques et numériques
}

Diane Pruneau, Jackie Kerry, Viktor Freiman, Joanne Langis et Janelle Cormier

\section{(2) OpenEdition}

12 Journals

Édition électronique

URL : http://journals.openedition.org/ere/762

DOI : $10.4000 /$ ere.762

ISSN : $2561-2271$

Éditeur

Centr'ERE

Édition imprimée

Date de publication : 20 décembre 2016

ISSN : 1373-9689

Référence électronique

Diane Pruneau, Jackie Kerry, Viktor Freiman, Joanne Langis et Janelle Cormier, « Les rapports préoccupants des futurs enseignants avec les milieux physiques et numériques », Éducation relative à l'environnement [En ligne], Volume 13 - 2 | 2016, mis en ligne le 20 décembre 2016, consulté le 21 février 2020. URL : http://journals.openedition.org/ere/762 ; DOI : 10.4000/ere.762 


\title{
Les rapports préoccupants des futurs enseignants avec les milieux physiques et numériques
}

\author{
Diane Pruneau, Jackie Kerry, Viktor Freiman, Joanne Langis et Janelle \\ Cormier
}

1 Puisque les programmes de sciences et technologies du Québec et du NouveauBrunswick incluent un nombre limité d'objectifs d'éducation relative à l'environnement (Pruneau et coll., 2013), et puisqu'il n'existe aucun programme dédié spécifiquement à ce domaine d'apprentissage, le choix d'éduquer les élèves à l'environnement demeure facultatif dans plusieurs écoles primaires de ces provinces. Ce type d'éducation est pourtant essentiel pour habiliter les futures générations à entretenir une relation significative avec leur milieu, à résoudre des problèmes environnementaux et à adopter des modes de vie écoresponsables. Malheureusement, plusieurs enseignants du primaire perçoivent l'éducation relative à l'environnement comme une composante peu prioritaire des programmes scolaires (Marx et Harris, 2006). Comment expliquer ce désintéressement des enseignants? On peut se demander si, de nos jours, le contact des futurs enseignants avec le milieu physique est assez significatif pour que ces derniers choisissent d'éduquer leurs élèves à l'environnement. En effet, les enseignants en formation (futurs enseignants), apparentés par leur âge aux natifs numériques (Oblinger et Oblinger, 2005), ont, dans leur enfance, passé moins de temps que les générations précédentes à jouer à l'extérieur (Burdette et Whitaker, 2005), font un usage massif des TIC et se distinguent des générations précédentes par leur manière de vivre, de penser, de travailler et d'apprendre (Lardellier et BryonPortet, 2010). L'utilisation intensive des TIC par ces jeunes adultes distancerait-elle ceux-ci du milieu physique? En éducation relative à l'environnement, on estime que le vécu d'expériences significatives en milieu naturel ou bâti favorise l'intérêt pour l'environnement et, éventuellement, l'engagement (Chawla, 2006). Lorsqu'une personne s'identifie à son milieu, elle en parle positivement et tend à s'engager dans des actions environnementales. Donc, si un enseignant s'intéresse au milieu physique (au monde vivant, aux écosystèmes), il transmettra son engouement à ses élèves et leur 
servira de modèle. Toutefois, on connaît aujourd'hui très peu la relation des futurs enseignants avec les lieux naturels ou construits de leur environnement.

De plus, qu'en est-il de la relation des futurs enseignants avec les lieux numériques (comme l'Internet ou les outils technologiques en ligne)? Accusées par certains mais célébrées par d'autres, les technologies représentent aujourd'hui une activité quotidienne des jeunes, ainsi qu'un outil pour les aider à accomplir plusieurs tâches simultanément et rapidement (Barnes, Marateo et Ferris, 2007). Toutefois, l'expérience des futurs enseignants dans les lieux numériques est encore méconnue. Que font-ils exactement dans ces lieux? Pourquoi les fréquentent-ils? Les ressources des milieux numériques (information, réseaux sociaux) contribuent-elles à mieux informer ces jeunes adultes au sujet de l'environnement et à soutenir leur action collective? Notre recherche avait pour but de documenter leurs rapports avec les lieux physiques (naturels et aménagés) et numériques, et d'identifier les significations que ces environnements ont pour eux. Quels sont les lieux qu'ils fréquentent? Que font-ils sur Internet, dans la nature et en milieu aménagé ou bâti? Quels sont les lieux auxquels ils s'identifient? Quels sont les stimuli qu'ils recherchent dans ces endroits? À quels besoins ces lieux répondent-ils? La recherche répondait donc à des besoins de développement des savoirs en éducation à l'environnement et en apprentissage par les TIC.

3 Le présent article fait état de nos entretiens avec des étudiants au Baccalauréat en enseignement primaire, à l'Université de Moncton et à l'Université du Québec à Montréal, sur leur vécu dans les lieux numériques et physiques (milieux naturels et construits).

\section{Relations aux lieux physiques}

4 Les concepts d'attachement au lieu (Proshansky, Fabian et Kaminoff, 1983), d'identité écologique (Thomashow, 1995), de sensibilité environnementale (Pruneau, 1995) et d'identité communautaire (Hummon, 1992) décrivent divers aspects d'une relation privilégiée avec l'environnement naturel ou construit. L'attachement au lieu, que Chow et Healey (2008) définissent comme un phénomène complexe et multidimensionnel incluant divers aspects du lien au lieu et comprenant des émotions, des savoirs, des comportements et des actions en rapport avec le lieu, figure parmi les facteurs qui encouragent l'implication des gens dans l'action environnementale (Chawla, 2006). Le vécu d'expériences significatives en milieu naturel ou bâti et l'influence positive des proches engendrent une relation étroite avec le milieu de vie (Arnold, Cohen et Wardner, 2009). Les personnes s'attachent entre autres à des lieux pour les avantages perçus de ceux-ci ou leurs affordances selon l'expression de Gibson (1977). Plus un lieu offre des opportunités de confort, d'intimité, de sécurité, de sérénité (Altman et Low, 1992) ou de liens amicaux (Pretty, Chipuer et Brampston, 2003), plus grand est l'attachement (Rollero et De Piccoli, 2010). En ce qui concerne les affordances du milieu naturel, Pruneau, Gravel et Ouattara (2002) ont trouvé que des adolescents considéraient la nature comme un endroit pour se reposer, réfléchir, s'émerveiller, jouer, rêver et apprendre. Enfin, Cuba et Hummon (1993) ont noté que l'implication des personnes dans des actions pour améliorer un lieu, contribuait à renforcer leur relation à ce lieu.

5 Toutefois, dans les dernières décennies, les anthropologues ont observé une diminution graduelle de la qualité des relations humaines avec le milieu biophysique et la 
communauté (Pyle, 1992). Malmberg (1992) décrit ce changement en termes de perte d'identité, de faible conscience de l'espace et du temps, d'oubli du rythme de la vie et d'affaiblissement $\mathrm{du}$ sens $\mathrm{du}$ territoire. Il explique cette perte d'identité communautaire et écologique par la déperdition de la nature sauvage et par la vie urbaine rapide. Il est question d'extinction de l'expérience, c'est-à-dire d'un contact moins direct et peu fréquent avec les phénomènes et acteurs du milieu d'appartenance. La plupart des gens seraient moins exposés régulièrement aux couleurs, formes, sons et magie de paysages riches et vivants. Ce phénomène social est peut-être à l'origine de l'apathie et du manque de responsabilité face à l'environnement. On en connait toutefois très peu sur la qualité actuelle des rapports des jeunes adultes et en particulier des futurs enseignants, avec leur milieu physique (milieu naturel et aménagé).

\section{Relations aux lieux numériques}

6 La recherche sur les activités numériques des jeunes adultes est aussi embryonnaire. L'Internet est maintenant considéré comme un environnement où les personnes vivent, apprennent, se développent et socialisent (Tapscott, 1999). L'univers social des futurs enseignants comprend des lieux physiques et virtuels, leur vie réelle et en ligne se complétant mutuellement (Larsen et Ryberg, 2011 ; Park, Lee et Kim, 2012). L'Internet façonne l'identité personnelle des jeunes adultes car ceux-ci utilisent quotidiennement les TIC à des fins de communication, de divertissement ou d'information (Martinovic et Freiman, 2011). Les technologies, accusées par certains mais célébrées et dédramatisées par d'autres, sont devenues un besoin. Les jeunes partagent avec leurs réseaux sociaux leurs préférences, créations, jeux, opinions et émotions (Larsen et Ryberg, 2011). Les réseaux sociaux, qui nécessitent un investissement considérable de temps au quotidien, constituent une source toujours disponible de contacts humains et de support pour ces jeunes (Horst et coll., 2010 ; Yang et Brown, 2013) et en particulier pour les filles (Junco, 2013a). Connectés, les jeunes s'influencent mutuellement en partageant leurs expériences, idées ou en mettant ces expériences et idées à la disponibilité des autres sur Internet (Margitay-Becht et Herrera, 2012). Leur quête d'interactions sociales les incite à communiquer avec un auditoire diversifié et les expose à une panoplie d'idées et de différences culturelles, tout en leur donnant une perspective d'inclusion sociale (Dorman, 2000). Les jeunes font partie de groupes d'intérêt, en lien avec leurs activités dans la vie réelle : sports, production artistique ou communauté culturelle (MargitayBecht et Herrera, 2012). L'accès accru à l'information renforce leur autonomie et leur sens critique. Préoccupés par la liberté d'expression, les jeunes ont des opinions fortes (Tapscott, 1999) et sont plus décisifs (Oblinger et Oblinger, 2005). Ils ont aussi développé des capacités multitâches (Boubée, 2011), soutenant que ces capacités sont nécessaires pour accomplir tout ce qu'il y a à faire dans le temps disponible (Barnes, Marateo et Ferris, 2007). Démontrant une faible tolérance aux textes et conférences, ils préfèrent les courtes explications visuelles, les multimédias interactifs, les environnements 3D et les animations (Oblinger, 2008). Avides de stimulations, ils préfèrent l'apprentissage actif, se montrant de ce fait des sujets kinesthésiques et expérimentaux (Pletka, 2007). L'expérience numérique des jeunes adultes, encore méconnue, comporte toutefois des aspects négatifs: cyberdépendance, cyberharcèlement, surcharge d'information et de stimuli, manipulation des valeurs par la 
transmission et la répétition d'informations biaisées et diminution des habiletés de communication en face-à-face (Feiertag et Berge, 2008).

\section{Méthodologie}

$7 \quad$ Notre étude de cas exploratoire visait une première compréhension des relations que les futurs enseignants entretiennent avec les milieux numériques et physiques. L'approche méthodologique de la théorisation ancrée (Glaser, 2007) a été retenue afin de construire cette compréhension de façon méthodique et graduelle, en vérifiant constamment les résultats et les interprétations. L'observation participante, les entretiens individuels puis de groupe ont été utilisés dans le but de modéliser progressivement les rapports des futurs enseignants avec les deux types de lieux. Au début de la recherche, afin d'éviter aux chercheurs de prendre position pour ou contre les TIC, les trois chercheurs ont procédé à une clarification de leurs biais personnels. En effet, l'un des chercheurs, adepte du monde numérique, y passait quotidiennement $70 \%$ de son temps. La deuxième chercheure fréquentait le milieu naturel tous les jours et limitait consciemment son temps en ligne. La troisième chercheure, native numérique, contrôlait aussi ses visites de l'Internet mais de façon moins importante. Cette dernière sortait marcher presque tous les jours. La discussion sur les biais personnels avait pour but de favoriser une meilleure écoute des paroles des participants à la recherche et d'éviter les jugements de valeur à propos des lieux où les gens devraient se rendre et dans quelle proportion. L'observation participante, quant à elle, visait à nous imprégner quelque peu des lieux numériques fréquentés par les jeunes adultes (comme Facebook ou les blogues). La chercheure principale a donc fréquenté Facebook pendant un an pour en comprendre les plaisirs et les enjeux. En 2013, des entrevues individuelles ont d'abord été réalisées pour amorcer la compréhension du vécu des étudiants dans les deux types de milieux. Elles ont été menées auprès de 12 étudiants volontaires (11 filles, 1 garçon) du Baccalauréat en enseignement primaire de l'Université de Moncton, et de 14 étudiants volontaires (10 filles, 4 garçons) du même programme à l'Université du Québec à Montréal. Les villes de Moncton et de Montréal ont été choisies en raison des faibles coûts nécessaires pour déplacer les chercheurs sur les lieux de l'étude. Les deux universités reçoivent des étudiants provenant de milieux urbains et ruraux. Les deux villes fournissent des commodités pour accéder à des parcs urbains et des lieux pour les rencontres sociales (bien que les cafés et restaurants soient moins variés à Moncton). Les étudiants interviewés en étaient à leur troisième ou quatrième année de leur programme de formation en éducation, c'est-à-dire presqu'à la fin de leur parcours universitaire. La présence prédominante des femmes parmi les participants à la recherche s'explique par la composition habituelle des cohortes des programmes de baccalauréat en enseignement au primaire.

8 L'une des entrevues portait sur les lieux numériques fréquentés et l'autre sur les lieux physiques. Voici des exemples des questions d'explicitation (Vermersh, 2003) du guide d'entrevue sur les lieux numériques: Si tu veux bien, je vais te ramener dans ta journée d'hier. Où es-tu allé en ligne? Choisis une activité que tu as faite. Que faisais-tu? A quoi pensais-tu? Comment te sentais-tu? Y vas-tu souvent? Pourquoi? Qu'est-ce qui te fait plaisir à cet endroit? Où emmèneras-tu tes élèves avec les technologies? Es-tu engagé dans une action collective? Voici des exemples des questions du guide d'entrevue sur les lieux physiques : Hier, tu dis que tu es allé... Rappelle-toi ce qui se passait autour de toi. Qu'est-ce que 
tu faisais à cet endroit? Faisais-tu autre chose? Rappelle-toi à quoi tu pensais. Veux-tu m'en parler? Était-ce plaisant pour toi à cet endroit? Explique-moi. Vas-tu souvent à cet endroit? Pourquoi? Une analyse thématique (Paillé et Mucchielli, 2008) des verbatim des entrevues individuelles de Moncton et de Montréal a permis de dégager une première compréhension des rapports des futurs enseignants avec les deux types de milieux: lecture indépendante du corpus par trois chercheurs, identification et description de thèmes, rencontres de concertation entre les chercheurs. La théorie des affordances (avantages perçus des lieux, selon Gibson, 1977) a principalement inspiré l'analyse. Des tableaux ont ainsi été préparés pour identifier les lieux fréquentés par les étudiants, leurs activités dans ces lieux ainsi que les émotions et les affordances perçues dans ces lieux. Pour obtenir un aperçu de l'importance des divers thèmes émergents, des chiffres représentant les nombres d'étudiants ont été associés aux différents thèmes.

En 2014, pour donner un sens aux observations cumulées, deux entrevues de groupe, l'une sur les milieux numériques et l'autre sur les milieux physiques, ont été réalisées avec 10 étudiants volontaires du cours obligatoire de didactique des sciences, à l'Université de Moncton. À l'aide de présentations Power Point montrant les premiers résultats, les participants aux entrevues de groupe ont été invités à se prononcer sur la validité des résultats, tels qu'interprétés par les chercheurs (à la suite des entrevues individuelles) et à commenter et compléter ces résultats. Les propos des étudiants, qui ont corroboré et précisé les résultats des analyses des entrevues individuelles, ont été ajoutés aux tableaux de résultats pour expliciter ou illustrer ces derniers. Des arbres thématiques ont finalement été préparés pour synthétiser les résultats.

\section{Résultats : rapports des futurs enseignants aux lieux numériques}

10 Les lieux numériques sont visités quotidiennement, longuement et à plusieurs reprises. Les plus fréquentés ${ }^{1}$ par les futurs enseignants sont, en ordre décroissant : Facebook, Internet via Google, YouTube, Pinterest et Skype (ou Facetime).

11 Les constats de notre étude au sujet des affordances de Facebook recoupent les résultats récents de Tosun (2012), de Bicen et Cavus (2011), de Cheung, Pui-Yee et Lee (2011) et de Dogruer, Menevis et Eyyam (2011) sur le réseautage en ligne des étudiants universitaires. Facebook, très populaire et visité très souvent durant la journée par nos participants, est utilisé pour prendre connaissance des nouvelles (des amis et des événements), regarder des photos et des vidéos des amis et de soi-même, participer à des groupes thématiques (travail, loisirs), organiser des rencontres sociales, communiquer avec des personnes proches et éloignées, commenter ce que les autres ont mis en ligne et jouer à des jeux de groupe. On attribue à Facebook les affordances suivantes: partage de ses réussites, de beaux moments et de belles photos; planification d'activités avec les amis; contact augmenté avec diverses personnes; manifestations d'attention et d'encouragements (on calcule le nombre de J'aime); entraide pour les travaux scolaires et dans la vie; aide à la décision; rappel de bons souvenirs; loisir et distraction; outil pour s'informer sur des personnes inconnues. Facebook fascine par sa disponibilité, sa rapidité (pas besoin de se déplacer), sa variété et sa facilité d'accès. Facebook procure des émotions: plaisir, frustration, colère, curiosité et autres. On va sur Facebook par habitude et/ou par obligation. On s'y montre consommateur d'informations et rarement créateur (d'idées, d'images ou de modes d'utilisation). On y reste toutefois prudent et critique quant au temps passé dans ce 
programme, quant à ses entrées et quant au choix de ses amis. Toutefois, comme dans l'étude de Junco (2013b), les futurs enseignants éprouvent de la difficulté à nous indiquer le temps exact passé quotidiennement sur Facebook.

Le monde d'Internet via Google, quant à lui, est employé pour les raisons suivantes: faire de la recherche; trouver des informations personnalisées; s'orienter dans l'espace ; organiser des voyages et des sorties ; chercher des idées nouvelles ; accéder à des informations non disponibles à proximité ; rencontrer des gens; chercher de la musique et des films ; naviguer ; magasiner et effectuer des transactions financières. On lui attribue diverses affordances : facilitation du travail et des projets (vitesse d'accès et variété d'information); aide à l'écriture (orthographe); source de plaisir; évasion du quotidien et palliation de la solitude.

13 Pour sa part, YouTube sert à regarder des vidéos d'information ou populaires, et à écouter de la musique. On le perçoit comme un lieu pour se divertir, s'informer, apprendre à exécuter une tâche, écouter des chansons, commenter des films ou se faire connaître comme artiste. Pinterest est employé pour trouver des suggestions d'activités pédagogiques ou des idées en lien avec ses intérêts : recettes, vêtements... Pinterest représente inspiration et excitation en raison des nouveautés qu'on y retrouve. Skype ou Facetime sont finalement des lieux de rencontre face-à-face, employés surtout avec des amis ou membres de la famille à distance. On leur attribue la fonction d'avoir accès au non-verbal des conversations.

Les arbres thématiques des figures 1 à 4, illustrent notre compréhension des rapports des futurs enseignants avec les lieux numériques. Dans ces figures, on remarque que les TIC semblent répondre à des besoins de la vie contemporaine : besoin d'accomplir de nombreuses tâches rapidement, de plaisir, d'inclusion et d'accès à un monde globalisé. Dans la figure 1 par exemple, on remarque que les TIC représentent des réponses à des besoins de fonctionnement et de performance dans une société rapide, comportant une surcharge de tâches à accomplir. Pour réaliser rapidement les tâches quotidiennes, on y consulte son réseau d'amis, on y cherche des informations ou des idées, on y effectue des transactions financières et on utilise des cartes géographiques en ligne.

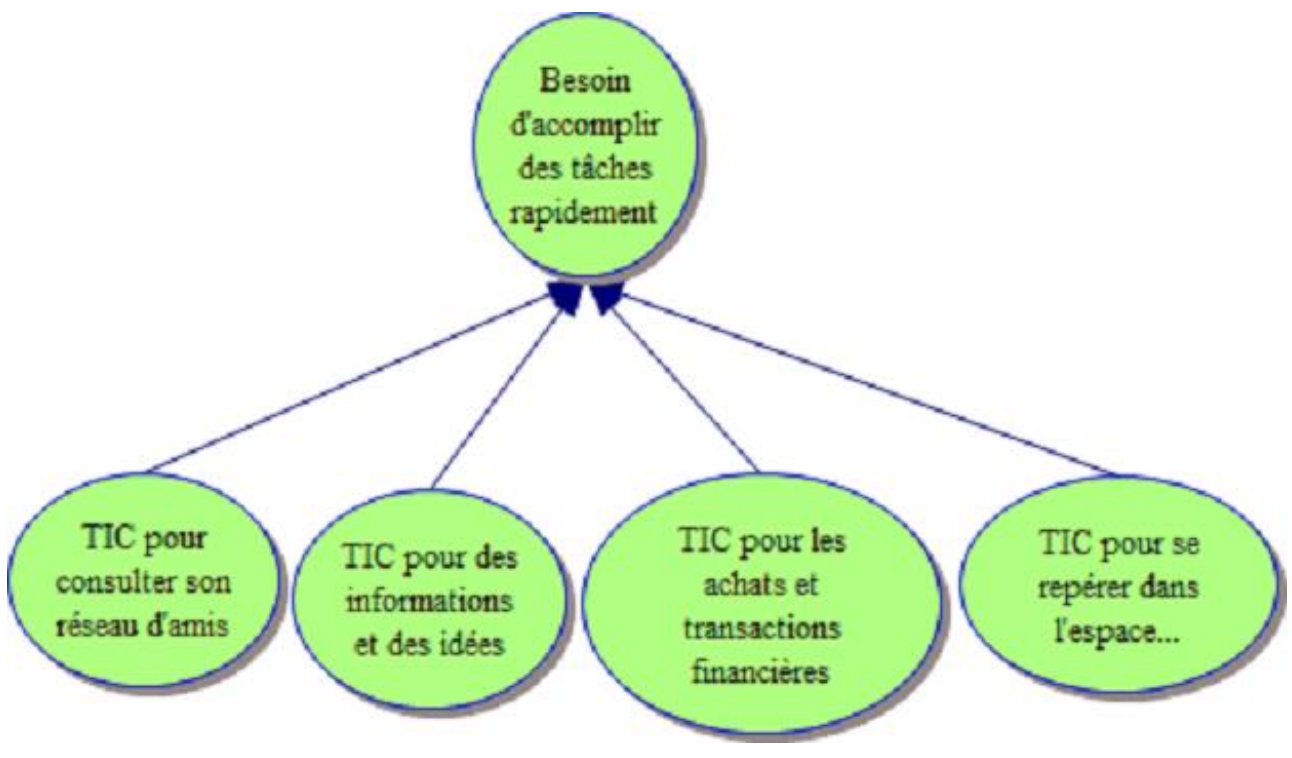


Figure 1 : Les TIC pour répondre aux besoins d'une vie rapide d'aventure. Pour nos répondants, il est plaisant de rencontrer des personnes nouvelles dans les TIC, d'y regarder de nombreuses choses présentées de manière à susciter la curiosité, d'y retrouver des éléments correspondant aux intérêts personnels, de les employer à la place des médias traditionnels plus coûteux comme la télévision ou le cinéma ou d'y jouer à des jeux.

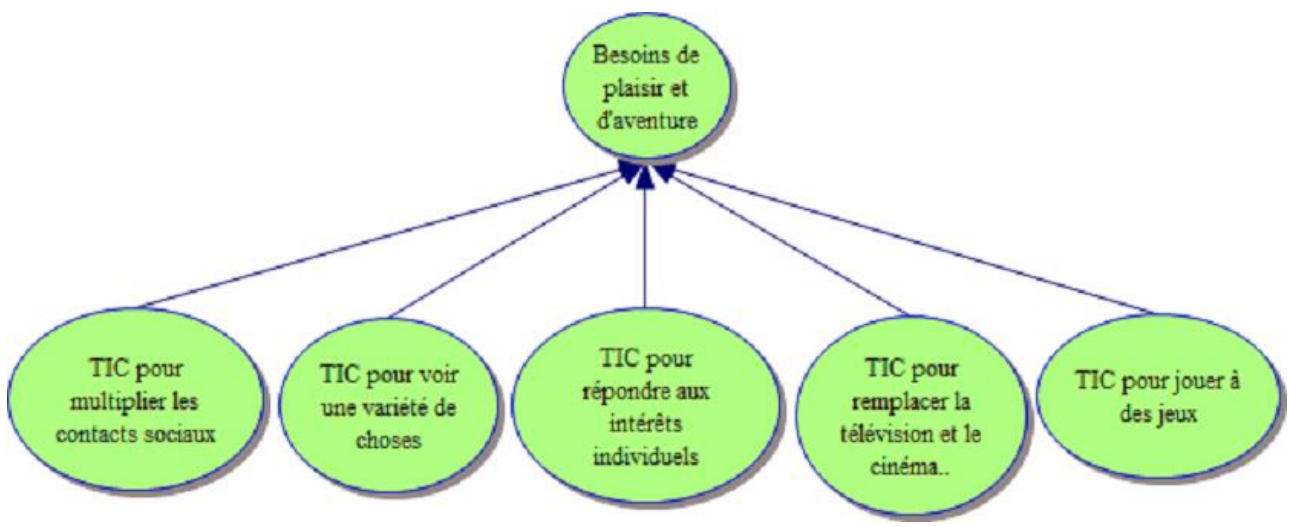

Figure 2 : Les TIC pour répondre aux besoins de plaisir et d'aventure

La figure 3 illustre le besoin d'inclusion sociale exprimé par nos répondants. Comme dans les études d'Akyildiz et Argan (2012) et de Nadkarni et Hofmann (2011), les TIC sont utilisés par nos répondants pour sentir qu'ils appartiennent à des groupes sociaux, pour montrer des aspects positifs d'eux-mêmes, pour combattre la solitude ou pour se faire de nouveaux amis.

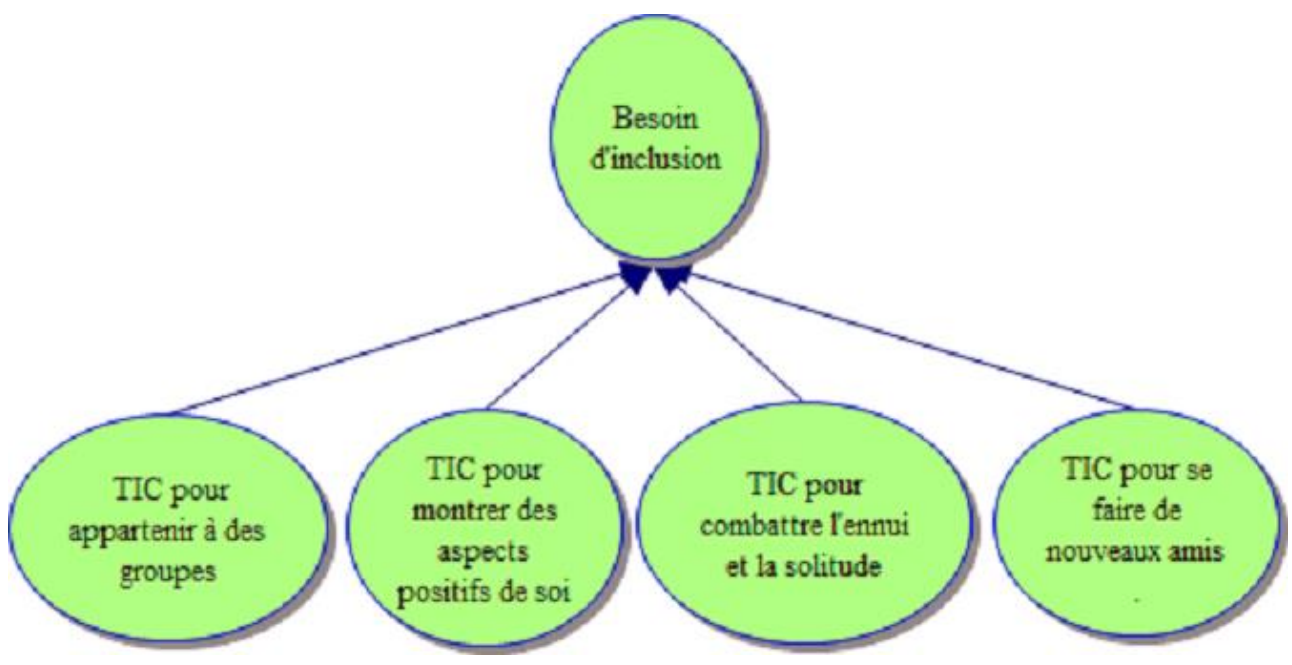

Figure 3 : Les TIC pour répondre au besoin d'inclusion

La figure 4 illustre enfin le besoin des futurs enseignants de participer à un monde globalisé et sans limite géographique. Nos répondants rêvent de voyager et d'entrer en contact avec d'autres cultures. Les TIC répondent à ces besoins en assurant le contact avec des paysages, des opinions et des cultures d'ailleurs. 


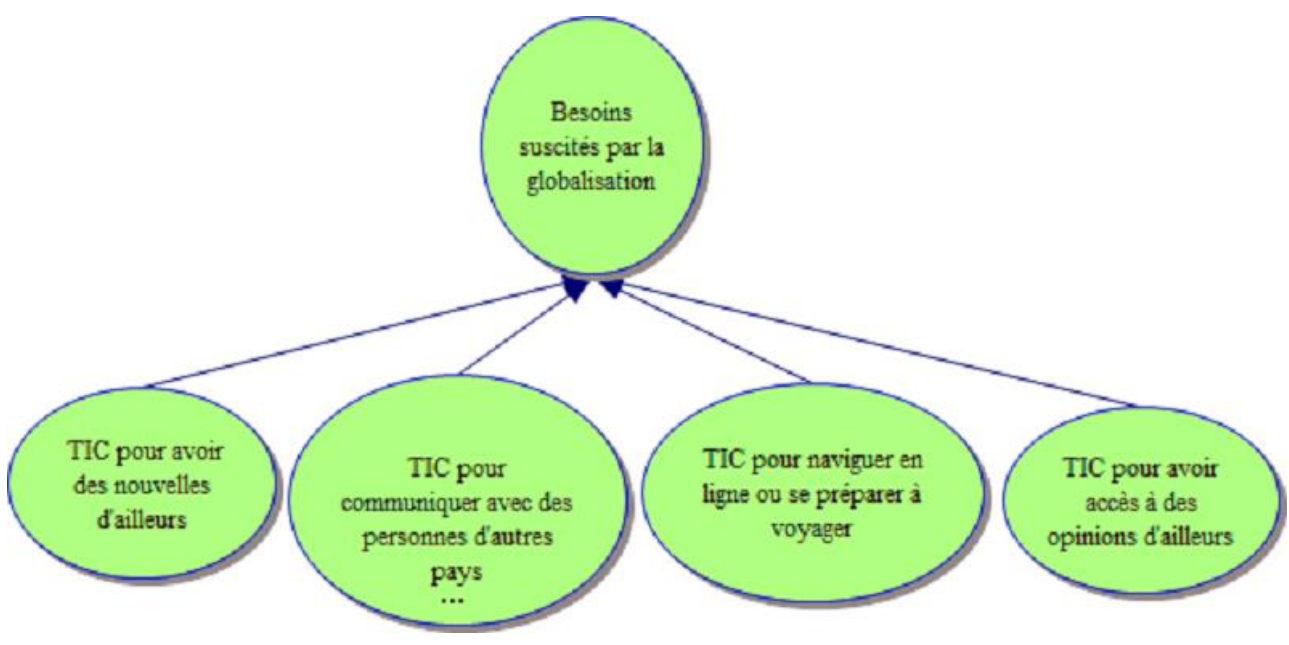

Figure 4 : Les TIC pour répondre aux besoins suscités par la globalisation idées, par exemple) ou pour s'engager dans des actions environnementales. En effet, les questions environnementales sont considérées davantage sous l'angle des problèmes que sous celui des opportunités. À part pour quelques répondants plus sensibles à la qualité du milieu de vie, le concept d'environnement est associé à une tâche de compréhension ou de résolution de problèmes complexes plutôt qu'à un engagement à améliorer la situation. Les futurs enseignants expriment enfin qu'une utilisation trop accentuée des TIC provoque certains jours des sentiments de fatigue et de saturation. Finalement, ils disent reconnaitre encore l'importance des relations humaines en faceà-face.

\section{Résultats : rapports des futurs enseignants aux milieux physiques}

Les lieux physiques, excluant les endroits de travail et d'études, sont visités plus sporadiquement que les lieux numériques. Leur fréquentation est limitée par la disponibilité du temps, par les saisons de l'année et par l'accès à des moyens de transport peu coûteux. Les lieux physiques les plus fréquentés par les futurs enseignants sont, en ordre décroissant : le milieu naturel et les parcs naturels urbains, les centres commerciaux, les maisons des amis, les centres sportifs, les restaurants/ cafés/bars, le cinéma et les paysages locaux par le biais de promenades en automobile.

En pleine nature et dans les parcs naturels urbains, les futurs enseignants, surtout ceux qui sont sportifs, font des activités physiques (vélo ou escalade, par exemple) ou de plein air (jardinage, camping, pique-nique, cueillettes, etc.). Ils marchent seul, avec des proches ou avec leur chien, souvent dans des sentiers qu'ils ont personnalisés. De plus, ils écoutent de la musique ou prennent des photos. Ils disent fréquenter les lieux naturels pour se garder en forme ou en santé, pour être bien avec leurs proches, pour contempler les éléments naturels, pour le silence, le calme et la relaxation (dépasser les tracas), pour réfléchir sur eux-mêmes, pour planifier leur avenir, pour se reposer du monde rapide et des TIC, ou encore pour se rappeler leur enfance (nostalgie). Dans la figure 5, on remarque que le milieu naturel représente une réponse à des besoins de retranchement d'une société rapide, de calme, de contemplation et de reconnexion avec soi. Les futurs enseignants se disent encore connectés au milieu naturel et 
conscients des avantages de ce type de lieu, même si leur fréquentation de ces endroits n'est pas constante (elle est plus accentuée à la belle saison).

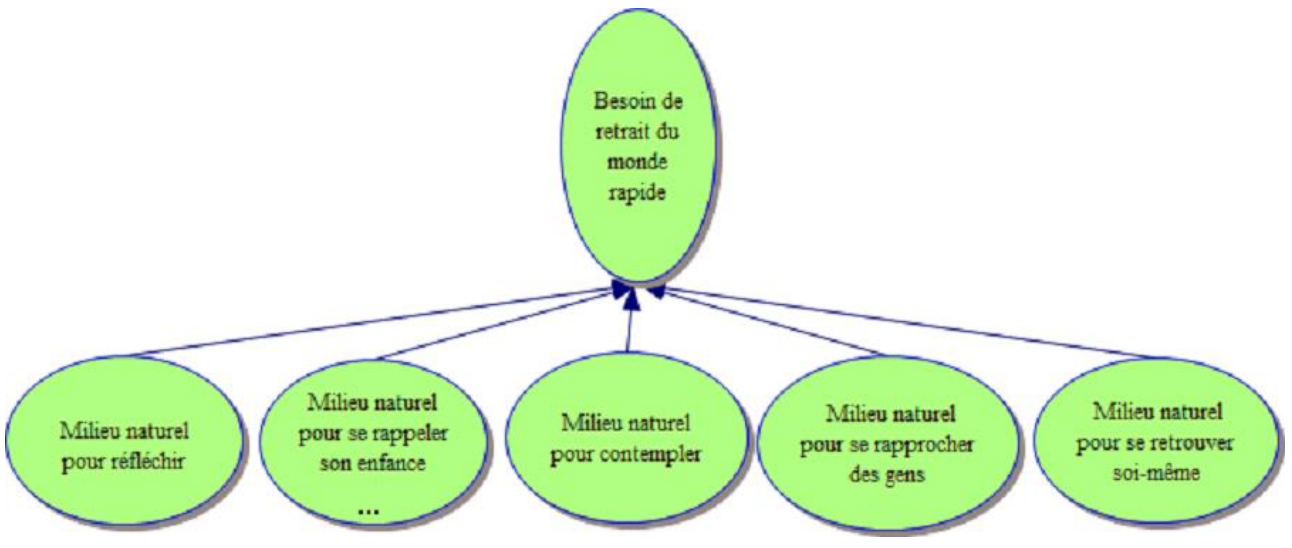

Figure 5 : Réponses du milieu naturel au besoin de retrait du monde rapide

Les futurs enseignants fréquentent les centres commerciaux pour faire des achats, se distraire, se changer les idées, voir les nouveautés ou rencontrer des personnes. Chez les amis, les répondants dansent, jouent à des jeux (de société, vidéos), parlent, travaillent, regardent des émissions ou préparent à manger ensemble. Les affordances associées aux visites chez des amis sont les suivantes : avoir des nouvelles et passer du temps de qualité ensemble. Quant à eux, les centres sportifs sont utilisés pour se garder en forme, pour oublier les tracas, pour les bienfaits de l'activité physique (humeur, estime de soi, énergie), pour rencontrer des personnes (élargir son réseau social), pour se dépasser physiquement ou pour se retrouver avec soi-même. Dans les cafés/bars/ restaurants, les futurs enseignants travaillent seuls ou en groupe, rencontrent des amis, mangent et écoutent de la musique. Ils associent les cafés/bars/restaurants à la complicité avec les amis, à la consommation d'aliments spécifiques ou nouveaux et à une ambiance propice au travail. Un autre type de lieu intéressant à mentionner et fréquenté uniquement par les futurs enseignants de Moncton est celui des promenades en automobile dans divers lieux situés à proximité. Dans leur village natal, les répondants de Moncton avaient adopté cette habitude de promenades en voiture. Seuls ou avec des amis, ils partent découvrir de nouveaux endroits de la région, regarder des paysages semblables à leur lieu d'origine, relaxer, réfléchir, éliminer les tracas ou se sentir proches de leurs amis ou d'un amoureux.

\section{Discussion}

Chez les futurs enseignants interrogés, âgés de 20-25 ans, les choix de fréquenter tel ou tel lieu numérique ou physique sont influencés par les habitudes et les expériences passées (par exemple, les promenades en automobile à Moncton), les plaisirs associés aux lieux, la saison, le grand nombre de tâches à accomplir (il faut faire vite), les choix de lieux des amis, le transport et les finances. Les lieux numériques sont principalement associés à de la stimulation (plaisir, surprise, curiosité) ou à la réalisation rapide de tâches. Les lieux naturels sont, quant à eux, associés au calme, au ressourcement et à la contemplation. Toutefois, pour les participants plus sportifs, les lieux naturels sont des endroits par excellence pour pratiquer l'activité physique. C'est ainsi que les affordances perçues des lieux numériques et des lieux naturels s'opposent 
catégoriquement. Les autres lieux physiques tels les centres commerciaux ou sportifs, les circuits en automobile et les résidences des amis servent à de multiples usages, surtout l'inclusion sociale et le plaisir.

Quand on questionne les futurs enseignants sur les lieux où ils emmèneraient leurs élèves, ils mentionnent nature, musée ou théâtre et, en ligne, programmes de traitement de textes, sites de recherche et YouTube. Il faut prendre en compte toutefois que les futurs enseignants ne peuvent, à ce point, être certains des choix d'environnements qu'ils feront pour leurs futures classes. En effet, diverses variables, encore peu présentes à leur esprit, influenceront leurs décisions dès leur entrée dans la profession: les objectifs des programmes scolaires, les opinions des parents au sujet des sorties éducatives, leur gestion du temps de classe, etc. Il reste toutefois que le milieu naturel leur semble actuellement détenir une valeur éducative ajoutée, même s'ils le fréquentent moins que durant leur enfance.

\section{Conclusion}

L'analyse des données de recherche a nécessité rigueur et prudence afin d'éviter les jugements de valeur quant aux choix de lieux des futurs enseignants. Il ressort que ceux-ci fréquentent très souvent des lieux numériques et parfois des lieux physiques. Ils semblent assez conscients des avantages et des limites des deux types de lieux. Leur usage des lieux numériques est toutefois critique pour la plupart d'entre eux. Plusieurs essaient volontairement de limiter le temps passé en ligne et de contrôler leur vécu sur Facebook. Leur usage des lieux naturels semble en diminution, même s'ils connaissent les avantages de ces lieux pour leur santé. Le temps consacré à répondre aux nécessités d'une vie rapide et la durée importante des moments passés dans les lieux numériques expliqueraient-ils la diminution de leur fréquentation du milieu naturel, malgré la valeur accordée à ce milieu ? La vie rapide et le manque de temps expliqueraient-ils leur faible implication dans l'action communautaire environnementale? Il nous semble que la valeur accordée au milieu naturel par les futurs enseignants, encore présente, s'estompe et se situe en arrière-plan dans leur conscience. Ils ont fréquenté ce milieu durant leur enfance, ils croient encore à ses effets bénéfiques mais des efforts semblent nécessaires pour que se matérialise leur intérêt pour ce milieu par des visites sur le terrain. Par contre, les étudiants plus sportifs semblent encore enclins à fréquenter le milieu naturel avec ardeur.

En termes d'attachement, les futurs enseignants du primaire qui, par tradition, sont surtout des femmes, se disent attachés à des amis, dont leurs amis Facebook. Cette plateforme d'échanges semble offrir des opportunités de confort, d'intimité, de sérénité, de connexion humaine et de plaisir, stimulant peut-être une identité communautaire à un groupe d'amis. Les futurs enseignants se disent de plus attachés aux lieux naturels de leur enfance mais moins aux lieux naturels et construits de leur ville actuelle de résidence. Leur identité communautaire parait ainsi se rattacher entre autres aux amis d'une " communauté » Facebook (ou autres partenaires numériques) et leur identité écologique, aux lieux de leur enfance.

Ces changements dans les identifications communautaires et écologiques posent des défis en ce qui concerne l'éducation aux lieux des futurs enseignants. Évoquant le manque de temps, plusieurs futurs enseignants interrogés disent se détacher progressivement du milieu naturel et de leur communauté de résidence, même s'ils 
reconnaissent les affordances de ces milieux. Encore pour des raisons de limite de temps, ils se disent peu impliqués dans des actions environnementales communautaires. Comment peut-on développer chez les futurs enseignants ces identités écologiques et communautaires qui sont à la base de l'engagement environnemental? Pendant les cours universitaires, nous croyons qu'il est essentiel d'emmener les futurs enseignants en milieu naturel, afin de raviver chez ces derniers la conscience des affordances de ce type d'endroit. Il importe aussi de les faire participer à des actions de restauration, d'embellissement ou de revitalisation de leur communauté de résidence. En effet, ils pourraient constater que leur implication dans des actions environnementales communautaires leur rapporte des avantages. Selon Schusler et Krasny (2010), Volk et Cheak (2003) et McClaren et Hammond (2005), les participants à de telles actions peuvent acquérir une conscience écologique, un intérêt pour les sciences, une préoccupation pour l'environnement, une estime de soi, des habiletés interpersonnelles, une autonomie, une identité communautaire, une attitude critique face au milieu et/ou un sentiment de pouvoir agir. De plus, si les actions sont réalisées en milieu naturel, les participants y gagneraient une appréciation pour la nature et un développement cognitif, social et émotionnel (Taylor \& Kuo, 2006). Des discussions critiques, durant les cours universitaires, aideraient également à ancrer ces expériences de vie. Les étudiants pourraient réfléchir à leurs choix de lieux, en fonction de leurs objectifs personnels de bien-être et de recherche de sens. Cette réflexion, les incitant à évaluer le potentiel éducatif de divers lieux, pourrait être élargie aux endroits où ils voudront emmener leurs élèves. Finalement, du coté numérique, il y aurait lieu de diversifier l'expérience des futurs enseignants en leur faisant explorer de nouvelles TIC: des outils numériques de travail collaboratif (Padlet, Popplet, RealtimeBoard, Stormboard et autres, qui sont des lieux de remue-méninges, de partage de perspectives et d'informations et de discussion), des outils numériques de représentation visuelle (par exemple, IDroo et SecondLife, pour la réalisation rapide de dessins et de modèles) et des outils numériques de publication (comme Glogster et Madmagz, qui offrent des possibilités de graphisme, d'images et de vidéos) (Pruneau \& Langis, 2015).

Des études complémentaires devront être réalisées afin de décrire les rapports aux lieux numériques et physiques des générations qui suivent celle de nos répondants. En effet, les futurs enseignants interrogés se disent inquiets par rapport aux étudiants universitaires plus jeunes qu'eux, qui n'ont pas eu la chance de jouer en nature, même s'ils habitaient à la campagne. Du côté des technologies, il y aurait lieu de poursuivre les recherches sur les activités des futurs enseignants reliées aux TIC en tenant compte de variables telles que le sexe, la personnalité, l'ethnie, l'âge et le statut marital des participants. Les récentes études de McAndrew et Jeong (2012) et de Seidman (2013) sur les usages de Facebook par les étudiants universitaires commencent à fournir certaines informations à ce sujet. De la même façon, il serait pertinent de comparer les lieux physiques fréquentés par les futurs enseignants démontrant et ne démontrant pas d'intérêt pour les activités sportives. Finalement, notre étude révèle que les frontières entre les lieux numériques et les lieux physiques sont parfois floues puisque les futurs enseignants utilisent les deux types de lieux en complémentarité. À titre d'exemple, Facebook est employé pour publier des photos d'une expérience significative vécue en milieu naturel ou Wikipedia est mis à contribution pour trouver le nom d'une plante intéressante aperçue en nature. L'étude de cette complémentarité entre les lieux 
physiques et numériques visités serait donc essentielle pour mieux situer dans l'espace réel et virtuel les activités et les préférences des futurs enseignants.

\section{BIBLIOGRAPHIE}

Akyildiz, M. et Argan, M. (2012). Using online social networking : Students' purposes of Facebook usage at the University of Turkey. Journal of Technology Research, 3 (1), 3-11.

Altman, I. et Low, S. M. (1992). Place attachment. A conceptual inquiry. New York : Plenum Press.

Arnold, H. E., Cohen, F. G. et Warner, A. (2009). Youth and environmental action : Perspectives of young environmental leaders on their formative influences. The Journal of Environmental Education, 40 (3), 27-36.

Barnes, K., Marateo, R.C. et Ferris, P.S. (2007). Teaching and learning with the Net Generation. Innovate Journal of Online Education, 3.

Bicen, H., et Cavus, N. (2011). Social network sites usage habits of undergraduate students : Case study of Facebook. Procedia - Social and Behavioral Sciences, 28, 943-947.

Boubée, N. (2011). Caractériser les pratiques informationnelles des jeunes : Les problèmes laissés ouverts par les deux conceptions. « Natifs » et «naïfs » numériques. Récupéré en mai 2014 du site : http:// www.cndp.fr/savoirscdi/fileadmin/fichiers_auteurs/Actes/Rennes_2011/NBRencontresSavoirsCDI-oct2011.pdf Burdette, H.L. et Whitaker, R.C. (2005). Resurrecting free play in young children. Archives of Pediatrics and Adolescent Medicine, 159, 46-50.

Chawla, L. (2006). Research methods to investigate significant life experiences : Review and recommendations. Environmental Education Research, 12, 359-374.

Cheung, K. M. C., Pui-Yee, C. et Lee, O. K. M. (2011). Online social networks : Why do students use Facebook? Computers in Human Behavior, 27 (4), 1337-1343.

Chow, K. et Healey, M. (2008). Place attachment and place identity : first year undergraduates making the transition to the university. Journal of Environmental Psychology, 28, 362-372.

Cuba, L. et Hummon, D. M. (1993). A place to call home : Identification with dwelling, community and region. The Sociological Quarterly, 34, 111-131.

Dorman, S.M. (2000). Implications of growing up digital. Review of Tapscott's Growing up digital. The Journal of School Health, 70, 420-422.

Dogruer, N., Menevi§, I. et Eyyam, R. (2011). What is the motivation for using Facebook ? Procedia - Social and Behavioral Sciences, 15, 2642-2646.

Feiertag, J. et Berge, Z.L. (2008). Training Generation N : How educators should approach the Net Generation. Education + Training, 50, 457-464.

Gibson, J.J. (1977). The theory of affordances. Dans R. Shaw and J. Bransford (dir.), Perceiving, acting and knowing (p. 142-159). Hillsdale, N.J. : Erlbaum.

Glaser, B.G. (2007). Doing formal grounded theory: A proposal. Mill Valley, CA : Sociology Press. 
Horst, H. A., Herr-Stephenson, B. et Robinson, L. (2010). Media ecologies. Dans M. Ito et coll. (Dir.), Hanging out, messing around and geeking out. Kids living and learning with new media (p. 29-78). Cambridge, Massachusetts : MIT Press.

Hummon, D.M. (1992). Community attachment. Dans I. Altman et S.M. Low (Dir.), Place attachment (p. 253-278). New York : Plenum Press.

Junco, R. (2013a). Inequalities in Facebook use. Computers in Human Behavior, 29, 2328-2336.

Junco, R. (2013b). Comparing actual and self-reported measures of Facebook use. Computers in Human Behavior, 29, 626-631.

Lardellier, P. et Bryon-Portet, C. (2010). Ego 2.0. Quelques considérations théoriques sur l'identité et les relations à l'ère des réseaux. Les Cahiers du Numérique, 1, 13-34.

Larsen, M. C. et Ryberg, T. (2011). Youth and Online social networking : From local experiences to public discourse. Dans E. Dunkels, G.-M. Franberg \& C. Hallgren (Dir.), Youth culture and Net culture: Online social practices (p. 17-40). New York : Information Science Reference.

Malmberg, T. (1992). Time and space in human ecology. Dans R.M. Dubey (Dir.), Human ecology and environmental education (p. 29-60). Allshabad, India : Chugh Publications.

Margitay-Becht, A. et Herrera, D. R. (2012). Immersion and connectivity : How goal-oriented fun can foster self-motivation. Communication présentée à la Conference Connectivity in the 21st Century, Salzburg, Austria.

Martinovic, D. et Freiman, V. (2011). Child and youth development beyond age 6 - Transitions to digitally literate adults. Communication présentée pour le Ministry of Child and Youth Services, Toronto, ON.

Marx, R. et Harris, C. (2006). No Child Left Behind and science education : Opportunities, challenges, and risks. Elementary School Journal, 106, 467-477.

McAndrew, T. F. et Jeong, S. H. (2012). Who does what on Facebook? Age, sex, and relationship status as predictors of Facebook use. Computers in Human Behavior, 28 (6), 2359-2365.

McClaren, M. et Hammond, B. (2005). Integrating education and action in environmental education. Dans E. A. Johnson et M. J. Mappin (Dir.), Environmental education and advocacy: Changing perspectives of ecology and education. (p. 267-291). Cambridge, UK : Cambridge University Press.

Nadkarni, A. et Hofmann, S.G. (2011). Why do people use Facebook? Personality and Individual Differences, 52 (3), 243-249.

Oblinger, D. (2008). Growing up with Google : What it means to education. Emerging Technologies for Learning, 3 (1), 11-29.

Oblinger, D. et Oblinger, J. (2005). Is it age or IT : First steps toward understanding the Net generation, Dans D.G. Oblinger et J.L. Oblinger (Dir.), Educating the Net Generation (p. 2.1-2.2). Boulder, CO : Educause.

Pletka, B. (2007). Educating the Net generation. Santa Monica, CA : Santa Monica Press.

Pretty, G., Chipuer, H. et Brampston, P. (2003). Sense of place amongst adolescents and adults in two rural Australian towns. Journal of Environmental Psychology, 23, 273-287.

Proshansky, H.M., Fabian, A.K. et Kaminoff, R. (1983). Place identity. Journal of Environmental Psychology, 3, 57-83. 
Park, N., Lee, S. et Kim, J.H. (2012). Individuals' personal network characteristics and patterns of Facebook use : A social network approach. Computers in Human Behavior, 28 (5), 1700-1707.

Pruneau, D. (1995). Conception et expérimentation d'un processus de formation en cours d'emploi destiné à inciter les enseignants du préscolaire et du primaire à agir en éducation relative à l'environnement. Thèse de doctorat, Québec : Université Laval.

Pruneau, D., Gravel, H. et Ouattara, I. (2002). Les relations que les adolescents entretiennent avec leur environnement. Revue des sciences de l'éducation, 28 (3), 565-586.

Pruneau, D., Kerry, M., Langis, J. et Léger, M. (2013). De nouvelles compétences à développer chez les élèves du primaire en sciences et technologies : pratiques et possibilités. Rapport de synthèse de connaissances pour le CRSH. Moncton, NB : Université de Moncton, Groupe de recherche Littoral et vie.

Pruneau, D. et Langis, J. (2015). Design thinking and ICT to create sustainable development actions. Proceedings of the 7th International Conference on Computer Supported Education, Lisbonne, Portugal, Volume 1, 442-446.

Pyle, R.M. (1992). Intimate relation and the extinction of experience. Left Bank, 2, 61-69.

Rollero, C. et De Piccoli, N. (2010). Place attachment, identification and environment perception : An empirical study. Journal of Environmental Psychology, 30, 198-205.

Schusler, T. M. et Krasny, M. E. (2010). Environmental action as context for youth development. The Journal of Environmental Education, 41 (4), 208-223.

Seidman, G. (2013). Self-presentation and belonging on Facebook : How personality influences social media use and motivations. Personality and Individual Differences, 54 (3), 402-407.

Tapscott, D. (1999). Educating the Net Generation. Educational Leadership, 56 (5), 6-11.

Taylor, A.F. et Kuo, F. E. (2006). Is contact with nature important for healthy child development? State of the evidence. Dans D. Spencer et M. Blades (Dir.) Children and their environments : Learning, using and designing Spaces. Cambridge : Cambridge University Press.

Thomashow, M. (1995). Ecological identity : Becoming a reflective environmentalist. Cambridge, Mass : MIT Press.

Tosun, P. L. (2012). Motives for Facebook use and expressing "true self" on the internet. Computers in Human Behavior, 28 (4), 1510-1517.

Vermersh, P. (2003). L'entretien d'explicitation. Paris : EME Éditions Sociales Françaises.

Volk, T. L. et Cheak, M. J. (2003). The effects of an environmental education program on students, parents, and community. Journal of Environmental Education, 34 (4), 12-25.

Yang, C. et Bradford Brown, B. (2013). Motives for using Facebook, patterns of Facebook activities, and late adolescents' social adjustment to college. Journal of Youth and Adolescence, 42 (3), 403-416.

\section{RÉSUMÉS}

Les futurs enseignants, dont plusieurs sont des natifs numériques, se distinguent des générations précédentes par leur manière de vivre et de travailler. L'utilisation des TIC contribue-t-elle à les distancer du milieu physique? Sont-ils engagés dans l'action environnementale? Notre étude visait à comprendre les rapports des futurs enseignants avec les milieux physiques et 
numériques. L'analyse des entrevues menées avec des étudiants en formation des maîtres, à Montréal et à Moncton, montre que les futurs enseignants entretiennent une relation discontinue avec le milieu naturel. Ils sont toutefois conscients que la nature leur procure calme, ressourcement et beauté. Fréquenté souvent, le milieu numérique leur offre distraction, affiliation sociale, information personnalisée, rapidité d'exécution et connexion avec un monde planétaire. Les futurs enseignants interrogés sont peu engagés en matière d'environnement. La recherche montre la nécessité de favoriser les rapports des futurs enseignants avec leur milieu de vie, à l'aide d'activités de terrain, de façon à les inciter à mieux connaître, apprécier et améliorer leur communauté écologique et sociale.

Future teachers among whom many are digital natives, can be distinguished from preceding generations by their way of life and work. Does the use of ICT contribute in distancing them from the physical environment? Are they involved in environmental action? Our study aimed at understanding the relationships future teachers have with the physical and digital environments. The analysis of interviews conducted with pre-service teachers in Montreal and in Moncton shows that future teachers have a fragmented relationship with the natural environment. However, they are aware that nature provides them calmness, rejuvenation and beauty. Often visited, the digital environment offers them distraction, social affiliation, personalised information, quick execution and connection with the global world. The interviewed future teachers have few committment to the environment. The research shows the necessity to promote future teachers' relationships with their living environment with the help of outdoor activities in order to encourage them to better know, appreciate and improve their ecological and social community.

\section{INDEX}

Mots-clés : TIC, action environnementale, enseignant, milieu naturel, milieu numérique, identité communautaire et identité écologique

Keywords : ICT, environmental action, teachers, natural environment, digital environment, community identity, ecological identity

\section{AUTEURS}

\section{DIANE PRUNEAU}

Diane Pruneau, professeure à la Faculté des sciences de l'éducation de l'Université de Moncton, dirige le Groupe de recherche Littoral et vie (www8.umoncton.ca/littoral-vie) qui effectue des interventions et recherches en éducation à l'environnement. Ses programmes de recherche nationaux et internationaux ont porté sur les relations des personnes avec leur environnement, sur l'éducation aux villes durables, sur l'éducation au changement climatique, sur le développement de compétences environnementales et sur l'emploi de la pensée design en environnement.

\section{JACKIE KERRY}

Jackie Kerry est chercheuse pour le Groupe de recherche Littoral et vie, de l'Université de Moncton. Elle travaille entre autres sur les compétences du 21e siècle et sur les compétences de viabilité. 


\section{VIKTOR FREIMAN}

Viktor Freiman, professeur à la Faculté des sciences de l'éducation de l'Université de Moncton, dirige le projet CompéTICA (Compétences en TIC en Atlantique). Il s'intéresse aux pratiques éducatives innovantes visant le développement du plein potentiel de chaque élève, à la résolution de problèmes dans un environnement virtuel et au développement de compétences numériques. Il a développé plusieurs ressources d'apprentissage en ligne, dont le site CAMI (Communauté d'apprentissages multidisciplinaires interactifs).

\section{JOANNE LANGIS}

Joanne Langis est coordonnatrice de projets pour le Groupe de recherche Littoral et vie, de l'Université de Moncton. Ce Groupe effectue des interventions pédagogiques en éducation relative à l'environnement auprès d'enseignants, d'élèves et de citoyens de la communauté. Ce Groupe réalise entre autres des programmes d'éducation aux actions environnementales en milieu urbain, à l'adaptation au changement climatique, à l'observation scientifique et aux gestes de protection contre le cancer.

\section{JANELLE CORMIER}

Janelle Cormier, étudiante de premier cycle au baccalauréat combiné en éducation secondaire et en sciences de l'Université de Moncton, travaille comme assistante de recherche pour le Groupe de recherche Littoral et vie. 